\title{
Emission line profiles as a probe of physical conditions in planetary nebulae
}

\begin{abstract}
Yong Zhang
Department of Physics, University of Hong Kong, Hong Kong

e-mail: zhangy96@hkucc.hku.hk

Received 10 January 2008 / Accepted 9 April 2008

ABSTRACT

Aims. We present an analysis of physical conditions in planetary nebulae (PNe) in terms of collisionally-excited line (CEL) and optical-recombination line (ORL) profiles. We aim to investigate whether line profiles could be used to study the long-standing CEL/ORL abundance-discrepancy problem in nebular astrophysics.

Methods. Using 1D photoionization models and their assumed velocity fields, we simulate the line profiles of various ionic species. We attempt to use our model to account for the observed CEL and ORL profiles. As a case study we present a detailed study of line profiles of the low-excitation planetary nebula (PN) IC 418.

Results. Our results show that the profiles of classical temperature and density diagnostic lines, such as [O III] $\lambda \lambda 4363,5007$, [S II] $\lambda \lambda 6716,6731$, and [Ar IV] $\lambda \lambda 4711,4740$, provide a powerful tool to study nebular temperature and density variations. The method enables the CEL/ORL abundance-discrepancy problem to be studied more rigorously than before. A pure photoionization model of a chemically-homogeneous nebula seems to explain the observed disagreements in the profiles for the [O III] $\lambda 4363$ and the $\lambda 5007$ lines, but cannot account for the differences between the [O III] CELs and the O II ORLs. We also investigate the temperature and density variations in the velocity space of a sample of PNe, which are found to be insignificant.
\end{abstract}

Key words. ISM: planetary nebulae: general - line: profiles

\section{Introduction}

As descendants of AGB (asymptotic giant branch) stars, planetary nebulae (PNe) serve as crucial probes of the chemical evolution of galaxies and stellar nucleosynthetic processes. However, the physical conditions inside PNe are not yet completely understood. An important problem in nebular astrophysics is that the heavy-element abundances measured from collisionally-excited lines (CELs) are systematically lower than those determined from optical-recombination lines (ORLs) (see Liu 2006a, for a recent review). The most extreme known case is that of Hf 2-2 which has an abundance discrepancy-factor (ADF) of about 70 (Liu et al. 2006). Possible explanations include electron temperature, electron density, and chemical-abundance inhomogeneities (Peimbert 1967; Viegas \& Clegg 1994; Liu et al. 2000), implying that PNe have complex physical conditions.

Many efforts have been made to develop new methods to probe nebular physical conditions. The traditional methods of using strong forbidden lines as plasma diagnostics suffer some disadvantages. Because of their low critical densities $\left(\$ 10^{5} \mathrm{~cm}^{-3}\right)$, CELs are strongly suppressed by collisional deexcitation in high-density regions, and cannot be used to probe the physical conditions inside dense clumps within the nebulae. Given that the fluxes of CELs have an exponential dependence on electron temperature, they also cannot be used to probe extremely-cold nebular regions where strong ORLs may arise. Improvements in the quality of observations and increasingly-accurate atomic data are allowing us to use ORLs as plasma diagnostics of PNe. Zhang et al. (2004) presented a method to obtain nebular electron temperature and density simultaneously using hydrogen-recombination spectra. Applying the method to 48 Galactic PNe, they found that the densities derived from hydrogen-recombination spectra were generally higher than those derived from forbidden line ratios and that the temperatures deduced from hydrogen-recombination spectra, $T_{\mathrm{e}}(\mathrm{H} \mathrm{I})$, were systematically lower than those derived from the $[\mathrm{O}$ III $]$ nebular-auroral line-ratios, $T_{\mathrm{e}}([\mathrm{O} \mathrm{III}])$, suggesting that temperature and density variations are generally present in $\mathrm{PNe}$. Zhang et al. (2005a,b) used He I recombination line-ratios to determine nebular electron temperatures, $T_{\mathrm{e}}(\mathrm{He} \mathrm{I})$, and found that $T_{\mathrm{e}}(\mathrm{He} \mathrm{I})<T_{\mathrm{e}}(\mathrm{H} \mathrm{I})$, supporting the hypothesis that some cold hydrogen-deficient clumps are embedded in diffuse gas with a "normal" chemical composition, as proposed by Liu et al. (2000) to explain the CEL/ORL abundance discrepancies. Some researchers presented nebular temperatures and densities derived from faint O II recombination-lines (Liu 2006b; Peimbert \& Peimbert 2005, and references therein). Their results suggest that $\mathrm{O}$ II recombination-lines may arise from colder and denser regions compared to the diffuse nebular gas.

Line profiles of PNe are of primary importance to investigate nebular physical conditions. To understand nebular dynamical evolution and geometry, Gesicki et al. (2003) and Morisset \& Stasińska (2006a) studied velocity profiles of a few strong emission lines, in terms of $1 \mathrm{D}$ and 3D photoionization models. A catalog of emission line profiles for PNe with various morphologies was presented by Morisset \& Stasińska (2006b, 2008). Emission line profiles can also provide an opportunity to test whether CELs and ORLs originate in different zones. To complete such a test, nebular spectroscopic data of high resolution and signal-tonoise are required. Ruiz et al. (2003) and Peimbert et al. (2004) analysed echelle spectra of the PNe NGC 5307 and NGC 5315. They found that O II ORLs and [O III] CELs had similar widths, implying that both lines originated in the same volume and disfavouring the scenario of chemical inhomogeneities. However, high spectral-resolution observations of two PNe NGC 6153 and 
NGC 7009 which have large CEL/ORL abundance discrepancies demonstrated that $\mathrm{O}$ II ORLs are significantly narrower than [O III] CELs (Barlow et al. 2006; Liu 2006a), implying that the ORLs and CELs originate in different zones. The spectra of NGC 6153 obtained by Barlow et al. (2006) show that the [O III] $\lambda 4363$ and $\lambda 5007$ lines have remarkably-different profiles, indicating the presence of large temperature variations within the nebula.

In this paper, we simulate nebular emission line profiles using photoionization models. Our main objective is to investigate whether line profiles could be used to study nebular physical conditions and the problem of ORL/CEL abundancediscrepancies in PNe. This paper is organized as follows. Section 2 presents our method. In Sect. 3, we use line profiles to study temperature and density variation within nebulae and compare with observations. Discussions and conclusions are presented in Sect. 4.

\section{Method}

The basic method is similar to that used by Gesicki et al. (2003) and Morisset \& Stasińska (2006a). We first construct photoionization models using the CLOUDY code (Ferland et al. 1998). A black-body spectral energy distribution is adopted for the ionizing stars. The nebulae are assumed to be a spherical shell with a given radial density distribution. CLOUDY calculates the emissivity distribution of the CELs and ORLs for different ionic species and the thermal structure of nebulae. Using known slit aperture and positions and based on an assumed radialvelocity field, we deduce the integrating line profiles by summing along the line of sight. For our modellings, we assume that the slit width is far narrower than the nebular radius since highresolution spectra are, in all cases, essential for the study. The predicted line profiles are then derived by convolving the broadening caused by temperature, seeing conditions, and instrument.

Turbulence is not taken into account in our modelling. Gesicki \& Zijlstra (2003) studied the expansion-velocity fields of a number of PNe and found that turbulence might be significant in Wolf-Rayet PNe. Based on a 3D modelling, Morisset \& Stasińska (2006a), however, argued that no turbulence is required to explain the observed line profiles if one considers a nebular-model departure from the assumption of spherical symmetry. It is therefore safe to assume that the effects of turbulence, on a normal PN, can be neglected.

Figure 1 provides an illustration of our modelling of a medium-excitation PN. The velocity field is assumed to have a positive gradient across the nebula. An inspection of Fig. 1 shows that the high-ionization lines are narrower than the lowionization lines. This is because the high-ionized species are mainly located within the internal regions where the radial velocity is lower. The profiles of emission lines arising from species with different ionization potentials have different dependencies on the velocity fields, and provide constraints of the nebular dynamics. Gesicki et al. (1996, 1998) and Gesicki \& Zijlstra (2000, 2003) investigated velocity fields for a sample of PNe by fitting the strong $\mathrm{H} \mathrm{I},[\mathrm{N} \mathrm{II}]$, and [O III] line profiles. They found that the expansion velocities generally increase outwards, consistent with the predictions of hydrodynamical calculations. Deep spectroscopic observations enable weaker lines including CELs and ORLs which cover a wider range of ionization potentials to be detected, and tighter constrains on the determination of velocity fields to be made. In the deep echelle spectra of the young PN IC 418, Sharpee et al. (2004) detected profiles of a number of CELs and ORLs and found a negative
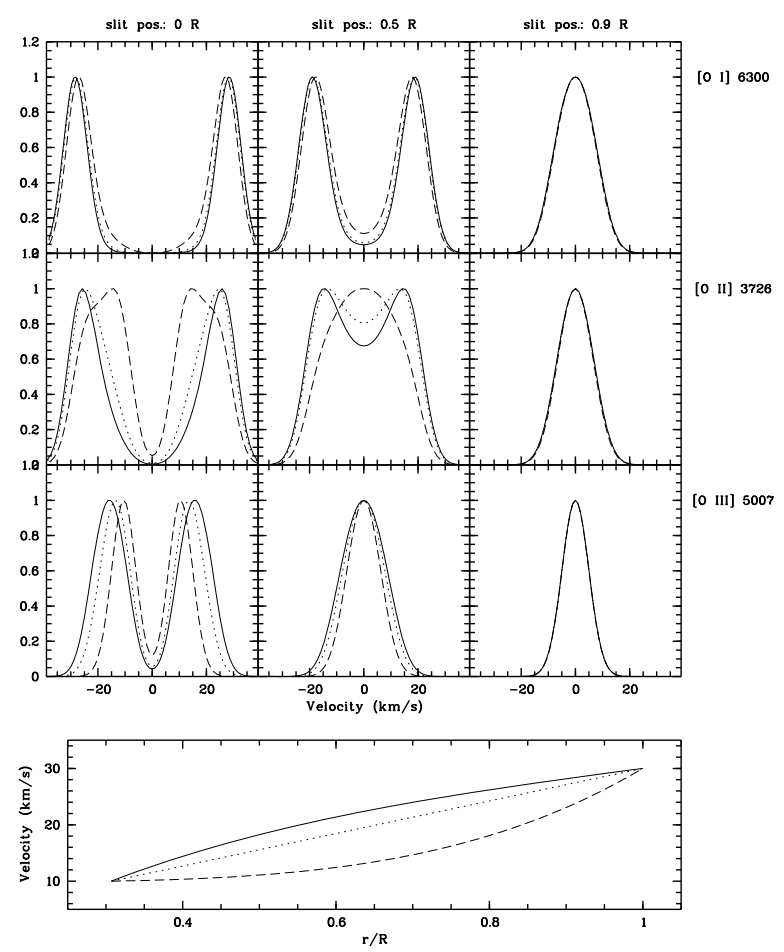

Fig. 1. Modelled [O I] (first row), [O II] (second row), and [O III] (third row) line profiles for a nebula observed by a long-slit located across the nebular centre (first column), half of the nebular shell (second column), and the nebular edge (third column). The peak flux of each line is normalized to unity. Solid, dashed, and dotted lines correspond to three different velocity fields shown in the lower panel.

correlation between line widths and ionization potentials. This is an implication that nebular expansion-velocities increase outwards. Throughout this paper, a monotonously-increasing velocity field is adopted.

For the model shown in Fig. 1, the [O II] line profiles are more sensitive to the velocity field than the [O I] and [O III] line profiles because the $\mathrm{O}^{+}$species are the dominant ionizationstate for the modelled medium-excitation nebula. Figure 1 also shows that the line widths decrease when the slit is placed towards the nebular edge. This tendency is particularly evident for low-ionization lines originating in external nebular regions. For a given expanding shell, this tendency is present because the velocity projected along the line of sight decreases from nebular centre to edge, and velocity broadening becomes less important with distance from the nebular centre. At the outer edge of nebulae, thermal broadening is dominant. Therefore, the emission lines originating in the outer edge of nebula have a Gaussian profile which is essentially independent of expansion velocity and only depends on electron temperature and atomic mass, as shown in Fig. 1.

\section{Analysis}

\subsection{Emission line profiles as a probe of temperature variations}

\subsubsection{Modellings}

Two scenarios were proposed to account for the ORL/CEL abundance discrepancies: 1) temperature fluctuations within the nebula (Peimbert 1967); 2) the two-component nebular model with extremely-cold hydrogen-deficient inclusions embedded in the 
diffuse nebula (Liu et al. 2000). The investigation of nebular temperature structure is vital for the solution of this problem. To quantitatively characterize the temperature variations within PNe, Peimbert (1967) introduced the average temperature $T_{0}$ and the mean square temperature fluctuation parameter $t^{2}$, defined by

$T_{0}=\frac{\int T_{\mathrm{e}} N_{\mathrm{e}} N_{\mathrm{i}} \mathrm{d} V}{\int N_{\mathrm{e}} N_{\mathrm{i}} \mathrm{d} V}$,

and

$t^{2}=\frac{\int\left(T_{\mathrm{e}}-T_{0}\right)^{2} N_{\mathrm{e}} N_{\mathrm{i}} \mathrm{d} V}{T_{0}^{2} \int N_{\mathrm{e}} N_{\mathrm{i}} \mathrm{d} V}$,

respectively, where $N_{\mathrm{i}}$ is the number density of the ionic species used to determine the electron temperature. For a nebula with small temperature fluctuations, $T_{0}$ and $t^{2}$ can be deduced by comparing $T_{\mathrm{e}}([\mathrm{O} \mathrm{III}])$ and $T_{\mathrm{e}}(\mathrm{H} \mathrm{I})$. By studying a large number of PNe, Zhang et al. (2004) found, however, that the average $t^{2}$-value of the nebulae was higher than predicted by photoionization models. This is probably evidence of the presence of extremely-cold ( $\$ 1000 \mathrm{~K})$ clumps in which ORLs are significantly enhanced and CELs are barely excited. To study nebular temperature fluctuations, Rubin et al. (2002) obtained a map of the $T_{\mathrm{e}}([\mathrm{O}$ III] $])$ of the PN NGC 7009 on the basis of high spatialresolution Hubble Space Telescope images. The spatial variation of $T_{\mathrm{e}}([\mathrm{O} \mathrm{III}])$ in a number of PNe was also studied by Krabbe et al. (2005). They found that the value of $T_{\mathrm{e}}$ ([O III] $)$ remains almost uniform across the PNe. Nevertheless, their results cannot completely rule out the scenario of temperature fluctuations as the cause of ORL/CEL discrepancies since their measurements of $T_{\mathrm{e}}([\mathrm{O} \mathrm{III}])$ are an average along each line of sight and provide only a lower limit to $t^{2}$. A comparison of line profiles may provide an unique way to estimate the temperature fluctuations along the line of sight.

We study the effect of temperature variations across the nebula on the profiles of the [O III] $\lambda 4363$ and $\lambda 5007$ lines. Figure 2 shows the modelled results of a high-excitation $\mathrm{PN}$ in which $\mathrm{O}^{2+}$ is the dominant ionization state. This model assumes that the hydrogen density in the nebula is $n(\mathrm{H})=10^{4} \mathrm{~cm}^{-3}$ and that the central star has an effective temperature of $150000 \mathrm{~K}$ and a luminosity of $4 \times 10^{37} \mathrm{erg} \mathrm{s}^{-1}$. As shown in Fig. 2, we assume that the velocity field follows a Hubble law. The slit is assumed to be midway between the central star and outer edge of the nebula. For the models used throughout this paper, the average chemical composition of the Galactic PNe (Kingsburgh \& Barlow 1994) is assumed. As shown in Fig. 2, the predicted electron temperature first decreases along the radius and then slightly increases at the outer edge of the nebula. The low-temperature regions are tightly associated with the $\mathrm{O}^{2+} / \mathrm{O}$ abundance ratio. This is a natural consequence of the relatively strong [O III] nebular lines that provide the dominant means of cooling in this PN.

Figure 2 shows that the variations of [O $\left[\begin{array}{lll}\mathrm{O} & \mathrm{III} & \lambda 4363\end{array}\right.$ and $\lambda 5007$ line profiles are very sensitive to nebular thermal structure, and thus provide an opportunity to probe nebular temperature variations. In the low-velocity regions where the electron temperature is higher than the average value, the [O III] $\lambda 4363$ line has a higher normalized intensity than the [O III] $\lambda 5007$ line. This is because the auroral forbidden line has a higher excitation energy, providing higher weighting to high temperature regions.

Based on high spectral resolution $(R=150000)$ observations of the PN NGC 6153, Barlow et al. (2006) found that the
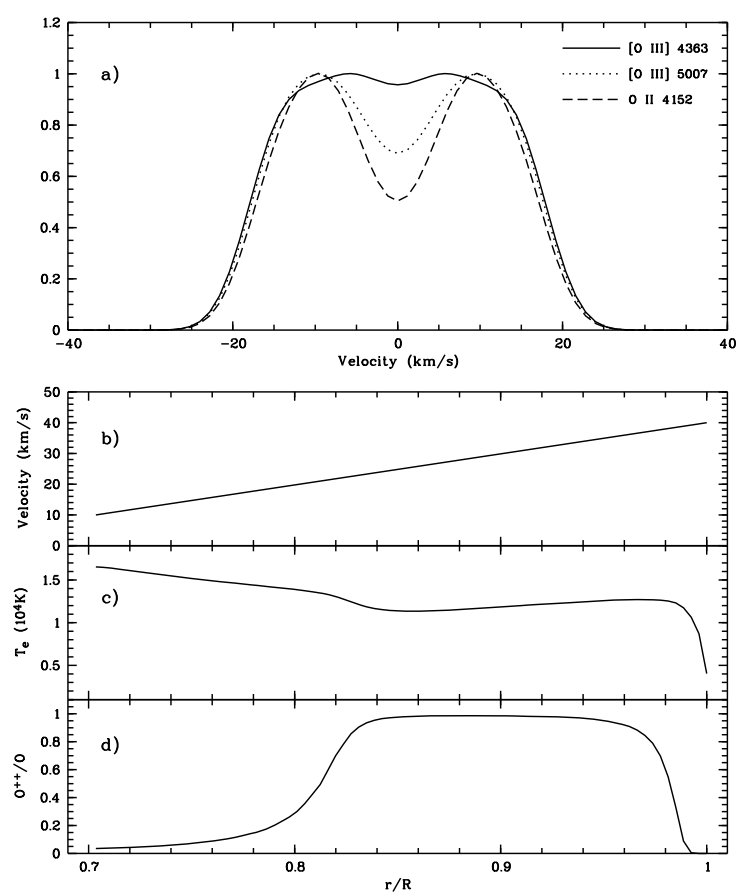

Fig. 2. Modelled results illustrating the effect of temperature variations on line profiles: a) the predicted line profiles of [O III] $\lambda 4363$, [O III] $\lambda 5007$, and O II $\lambda 4152$; b) the assumed velocity field; c) the radial temperature distribution; d) the radial $\mathrm{O}^{2+} / \mathrm{O}$ abundance-ratio distribution.

[O III] $\lambda 4363$ and $\lambda 5007$ lines have different profiles, that is, the gap between the two peaks of the $\lambda 5007$ line is filled in the case of the $\lambda 4363$ line. This can be explained in terms of our modelling, as shown in Fig. 2. It is interesting that the $t^{2}$ value $(\sim 0.01)$ required to match the observed [O III] $\lambda \lambda 4363,5007$ line profiles is much lower than that derived from the $\mathrm{H}$ I Balmer Jump and [O III] lines $\left(t^{2}=0.045\right.$, Liu et al. 2000). This appears to support the idea that the two-abundance nebularcomponent model accurately describes the observations, to discount the theory that temperature fluctuations are the cause of the CEL/ORL abundance dichotomy. These conclusions are, however, arguable since the analysis of [O III] $\lambda \lambda 4363,5007$ line profiles can provide only a lower limit to the temperature variations along the line of sight (see below).

\subsubsection{Temperature variations in the velocity space}

To study the temperature variations, we define the average temperature $T_{0, v}$ and the mean square temperature fluctuation parameter $t_{v}^{2}$ in velocity space by

$T_{0, v}=\frac{\int T_{\mathrm{e}}(v) I_{v}(\mathrm{H} \beta) \mathrm{d} v}{\int I_{v}(\mathrm{H} \beta) \mathrm{d} v}$,

and

$t_{v}^{2}=\frac{\int\left[T_{\mathrm{e}}(v)-T_{0, v}\right]^{2} I_{v}(\mathrm{H} \beta) \mathrm{d} v}{T_{0, v}^{2} \int I_{v}(\mathrm{H} \beta) \mathrm{d} v}$

where $v$ is the velocity along the line of sight, and $T_{\mathrm{e}}(v)$ and $I_{v}(\mathrm{H} \beta)$ are the electron temperature deduced from the forbidden line intensity ratio and the intensity of $\mathrm{H} \beta\left(\propto N_{\mathrm{p}} N_{\mathrm{e}}\right)$ at a given velocity, respectively. The integrations in the two equations are over velocity space. As defined by Eqs. (3) and (4), $T_{0, v}$ and $t_{v}^{2}$ 
Table 1. The values ${ }^{a}$ of $T_{0, v}$ and $t_{v}^{2}$.

\begin{tabular}{cccc}
\hline \hline Object & IC 2501 & IC 4191 & NGC 2440 \\
\hline$T_{0}(\mathrm{~K})$ & 7700 & 8600 & 12600 \\
$t^{2}$ & 0.053 & 0.040 & 0.077 \\
$T_{0, v}(\mathrm{~K})$ & 11500 & 10200 & 14800 \\
$t_{v}^{2}$ & 0.002 & 0.001 & 0.011 \\
\hline
\end{tabular}

${ }^{a}$ For the low-excitation PN, IC 2501, $T_{0, v}$ and $t_{v}^{2}$ are deduced using the [N II] $\lambda \lambda 5754,6678$ lines; for the high-excitation PNe, IC 4191 and NGC 2440, $T_{0, v}$ and $t_{v}^{2}$ are deduced using the [O III] $\lambda \lambda 4363,5007$ lines.

characterize temperature fluctuations along the line of sight and are similar to those introduced to explain the $T_{\mathrm{e}}([\mathrm{O} \mathrm{III}]) / T_{\mathrm{e}}(\mathrm{H} \mathrm{I})$ discrepancy, which are given in Eqs. (1) and (2). In Eqs. (3) and (4), we can use $I_{v}\left(\mathrm{X}^{i+}\right)$, which is the intensity of a CEL used to determine $T_{\mathrm{e}}(v)$, as weights instead of $I_{v}(\mathrm{H} \beta)$. However, if significant density variations are present along the line of sight, it may be problematic to use $I_{v}\left(\mathrm{X}^{i+}\right)$ since its density dependences differ in high- and low-density regions.

Using Eqs. (3) and (4), we calculated $T_{0, v}$ and $t_{v}^{2}$ for three PNe, IC 2501, IC 4191, and NGC 2440. Our data were acquired using the Las Campanas Observatory (LCO) Baade $6.5 \mathrm{~m}$ telescope with the MIKE echelle spectrograph, yielding a resolution of 25000 (see Sharpee et al. 2007, for a detailed description of the observations). The results are given in Table 1. For IC 4191 and NGC 2440 the values of $T_{0, v}$ and $t_{v}^{2}$ are measured using the [O III] $\lambda \lambda 4363,5007$ lines. For the low-excitation PN IC 2501, the slit was placed on the edge of the compact $\mathrm{O}^{2+}$ regions and the thermal broadening provides a dominant contribution to the profiles of the [O III] lines. For IC 2501 we obtained $T_{0, v}$ and $t_{v}^{2}$ using instead the single-ionization lines [N II] $\lambda \lambda 5754,6678$ lines. For the calculations, the reddening coefficient and the electron densities presented by Sharpee et al. (2007) were adopted. As depicted in Fig. 3, the temperature variations in the velocity space are minor for the three PNe.

Based on the values of $T_{\mathrm{e}}([\mathrm{O} \mathrm{III}])$ and $T_{\mathrm{e}}(\mathrm{H} \mathrm{I})$ presented by Sharpee et al. (2007), we determined $T_{0}$ and $t^{2}$ using Peimbert's formulae (Peimbert 1967). The calculations were based on the assumption that the $\mathrm{O}^{2+}$ and $\mathrm{H}^{+}$regions are identical, which is appropriate for our studied $\mathrm{PNe}$ since their $\mathrm{O}^{+} /\left(\mathrm{O}^{+}+\mathrm{O}^{2+}\right)$ abundance ratios are consistently lower than 0.1 (Sharpee et al. 2007). The resultant $T_{0}$ and $t^{2}$ are given in Table 1 . If the discrepancy between $T_{\mathrm{e}}([\mathrm{O} \mathrm{III}])$ and $T_{\mathrm{e}}(\mathrm{H} \mathrm{I})$ is caused by small temperature fluctuations, the $t^{2}$ measured by $T_{\mathrm{e}}([\mathrm{O} \mathrm{III}])$ and $T_{\mathrm{e}}(\mathrm{H} \mathrm{I})$ should be identical to those measured along the line of sight. However, Table 1 shows that the values of $t_{v}^{2}$ are consistently lower than those of $t^{2}$ and the values of $T_{0, v}$ are consistently higher than those of $T_{0}$, seemingly favoring the presence of extremely-cold nebular components where the [O III] lines cannot be excited but the $\mathrm{H}$ I recombination spectrum is significantly strengthened.

This conclusion, however, needs to be treated with caution. The value of $t_{v}^{2}$ defined by Eq. (4) represents the temperature variations in velocity space. We should bear in mind that the validity of the argument that $t_{v}^{2}$ is equal to the mean square temperature variation parameter along the line of sight requires two conditions to be satisfied: a) nebular expansion-velocity dominates the broadening of line profiles; b) the expansion-velocity is monotonic along the nebular radius, as found in most of PNe. Otherwise, $t_{v}^{2}$ provides only a lower limit to the temperature fluctuations along the line of sight. In any case, the comparison of $t_{v}^{2}$ and $t^{2}$ provides an opportunity to investigate the presence of extremely-cold components. This paper is beginning such a

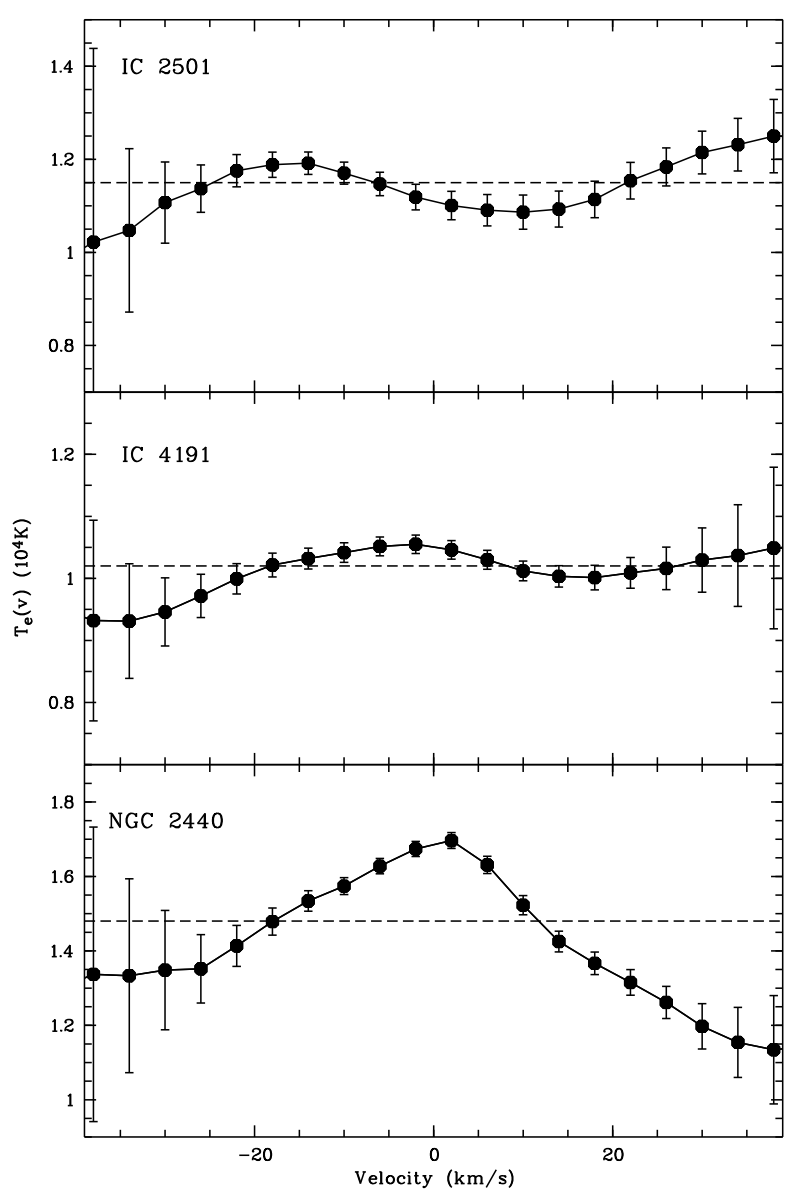

Fig. 3. Electron-temperature distribution in the velocity space of PNe, IC 2501, IC 4191, and NGC 2440. The dashed lines represent the values of $T_{0, v}$.

project. Higher-resolution spectroscopic data of PNe with a high expansion velocity will be invaluable for this study.

\subsubsection{ORL profiles versus CEL profiles}

Figure 2 also depicts that if temperature variations exist, the $\mathrm{O}$ II lines show a profile that differs from that of the [O III] lines, which can be attributed to the fact that CELs and ORLs weight high- and low-temperature regions, respectively. In the low velocity regions, the electron temperature is relatively high. As a result, the O II lines have a deeper "gap" between the two peaks than the $[\mathrm{O}$ III] lines. In the high velocity regions which correspond to the line wings, the electron temperature is slightly higher, depressing recombination lines. Consequently, the $\mathrm{O}$ II lines are narrower than the [O III] lines. The $\mathrm{O}$ II line profiles differ most significantly from those of the [O III] $\lambda 4363$ line than of the [O III] $\lambda 5007$ line, since the [O III] $\lambda 5007$ line has a lower excitation temperature than the [O III] auroral line.

High spectral-resolution observations of PN NGC 7009 show that the [O III] $\lambda 4363$ CEL is broader than the O II ORLs by a factor of about 1.5 (Barlow et al. 2006). For their observations, a $0.9^{\prime \prime} \times 0.9^{\prime \prime}$ image-slicer was placed on a bright-edge region located 5.6" northwest of the central star. We attempted to model the observed CEL/ORL width discrepancy. However, we find that chemically-homogeneous nebula models are unable to reproduce a CEL/ORL width ratio that is greater than 1.2 because our modellings are unable to achieve such large temperature 
variations. The modelled CEL/ORL width ratio is essentially independent of the adopted velocity field and is insensitive to the assumed density distribution since the [O III] $\lambda 4363$ CEL and the O II ORLs both have high critical densities. To match the observed CEL/ORL widths properly, the electron temperature is required to increase sharply outwards, which is difficult to achieve using pure photoionization modelling of gaseous nebulae. The most direct explanation for the observed large CEL/ORL width discrepancy is that the $\mathrm{O}^{2+}$ CELs and ORLs originate in different nebular components which have separate kinematic fields. Liu (2003) proposed that evaporating planetesimals within the nebulae might produce cold H-deficient inclusions where ORLs are greatly enhanced and CELs are depressed. If this is the case, these H-deficient components should move in relatively-stable orbits, and thus have a lower velocity dispersion than the diffuse nebular gas. As an alternative, it remains possible that heating by shock waves in the outer regions may be significant and leads to a sharp temperature increase in the high-velocity regions. Furthermore, Stasińska \& Szczerba (2001) showed that photoelectric heating by dust grains can cause large temperature fluctuations in the presence of density inhomogeneities. However, in the case of a constant dust-to-gas ratio in the entire nebula, the temperature is more efficiently enhanced in the inner regions. If the CEL/ORL width discrepancy is caused by heating by dust, these dust grains must be predominantly located in the outer zones of the ionized gas.

\subsection{Emission line profiles as a probe of density variations}

\subsubsection{Modellings}

The determination of nebular density structure is critical to the investigation of ORL/CEL abundance discrepancies. Viegas \& Clegg (1994) pointed out that the presence of high density regions could lead to an overestimate of $T_{\mathrm{e}}([\mathrm{O} \mathrm{III}])$ and consequently an underestimate of CEL abundances since the $\lambda 5007$ nebular line has a far lower critical density than the [O III] $\lambda 4363$ auroral line and is more significantly suppressed by collisional de-excitation in-high density regions. However, Liu et al. (2000) claimed that density inhomogeneities can be ruled out as the cause of the ORL/CEL abundance discrepancies because no correlation between the abundance discrepancies and critical densities was found. Line profiles can be used to probe density variations along the line of sight.

The [S II] $\lambda \lambda 6731,6716$ doublet lines are classical electrondensity diagnostics. We therefore simulate the profiles of the [S II] lines under the assumption that density decreases outwards and increases outwards, as shown in Figs. 4 and 5, respectively. We assume that the ionizing star has a temperature of $30000 \mathrm{~K}$. For the nebulae that we model, $\mathrm{S}^{+}$is the dominant ionization-state of sulphur. Figures 4 and 5 indicate that the the profiles of the [S II] $\lambda \lambda 6731,6716$ doublet lines differ if density variations are present. The [S II] $\lambda 6731$ line has a higher critical density of $\sim 4 \times 10^{3} \mathrm{~cm}^{-3}$ than the $\lambda 6716$ line $\left(\sim 1 \times 10^{3} \mathrm{~cm}^{-3}\right)$, and hence is less-affected by collisional deexcitation in high-density regions. For a density that decreases and increases outwards, the [S II] $\lambda 6731$ line is then stronger in low- and high-velocity regions, respectively. As shown in Figs. 4 and 5 , the profile discrepancy is particularly remarkable when the [S II] $\lambda \lambda 6731,6716$ lines and the [S II] $\lambda 4076$ line are compared because the latter has a much higher critical density $\left(\sim 10^{6} \mathrm{~cm}^{-3}\right)$. However, we note that temperature fluctuations may contribute partially to the profile discrepancy between these density diagnostic lines.
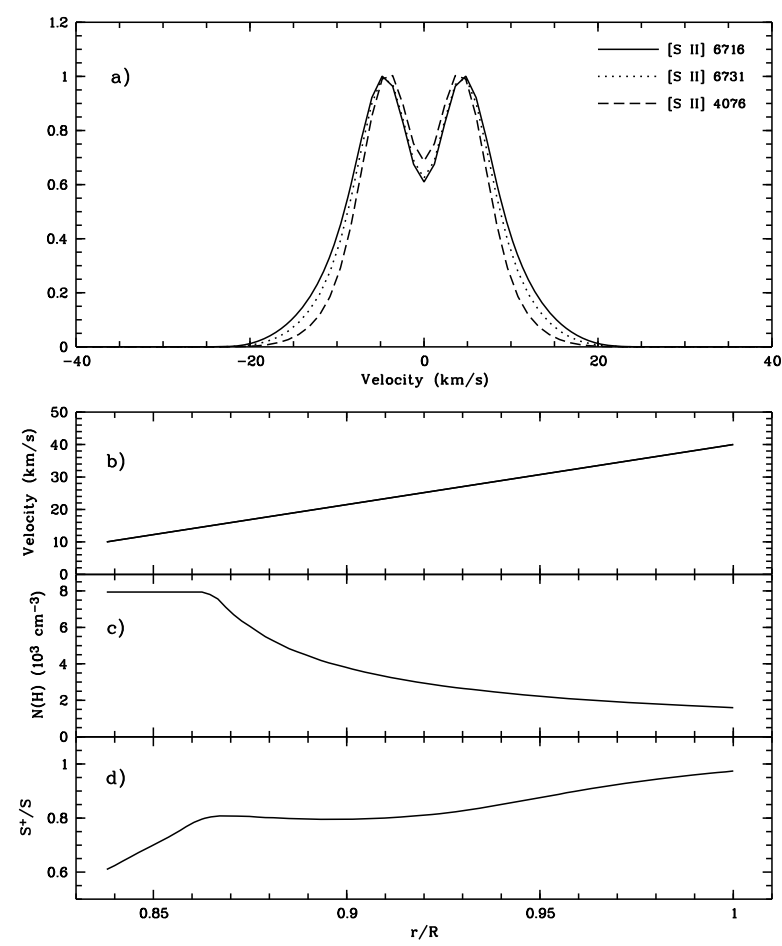

Fig. 4. Modelled results illustrating the line profiles for an outwardlydecreasing density distribution: a) the predicted line profiles of [S II] $\lambda 6716$, [S II] $\lambda 4731$, and [S II] $\lambda 4070$; b) the assumed velocity field; c) the radial density distribution of hydrogen atoms; d) the radial $\mathrm{S}^{+} / \mathrm{S}$ abundance-ratio distribution.
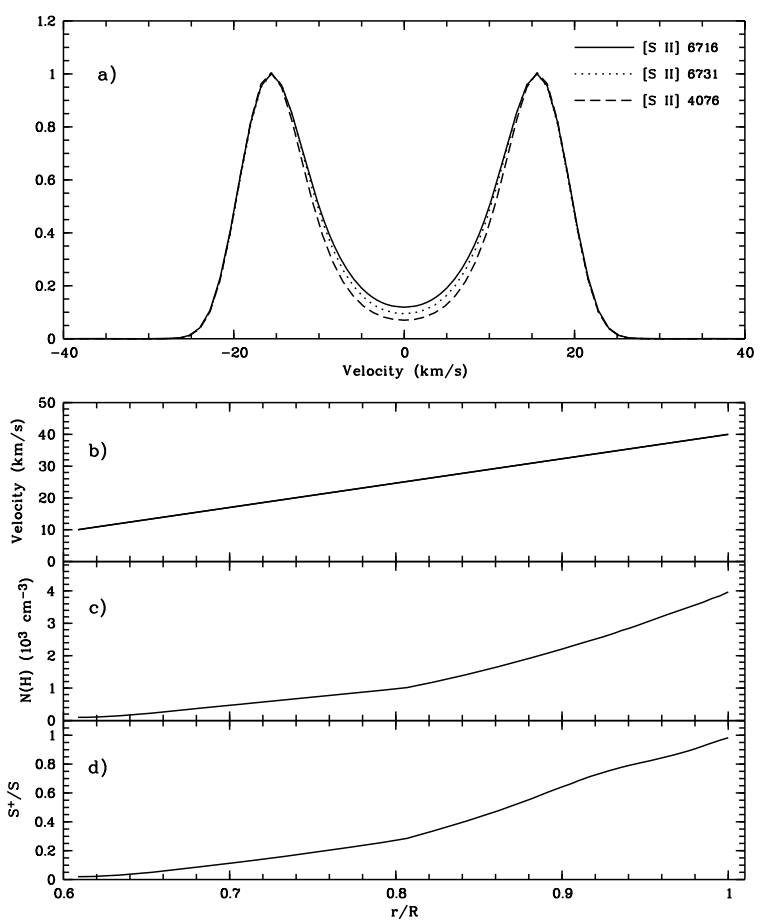

Fig. 5. Same as 4 but for an outwardly-increasing density distribution.

Apart from for the density distribution, the basic assumptions are identical in each model. Figure 5 shows a larger separation between the two emission peaks than in Fig. 4. This is because in the case of outwardly-increasing density distribution, emission lines weight the high-velocity regions more highly. 


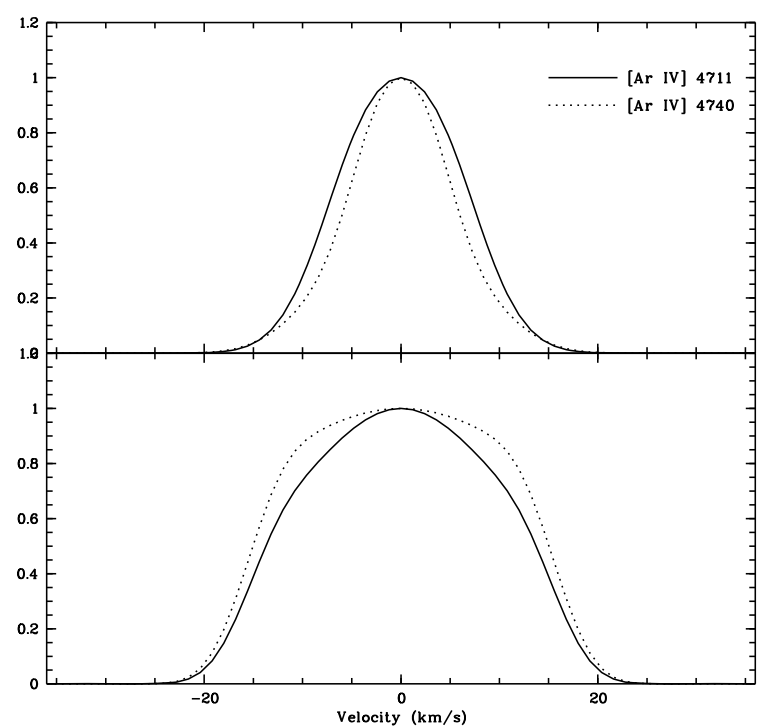

Fig. 6. The profiles of the [Ar IV] $\lambda \lambda 4711,4740$ lines for outwardlydecreasing (upper panel) and increasing (lower panel) density distributions.

The analysis methods are applied to other density diagnostics, such as [O II] $\lambda \lambda 3726,3729,[\mathrm{Cl}$ III] $\lambda \lambda 5517,5537$, and $[\mathrm{O}$ II] $\lambda \lambda 4711,4740$. As an example, Fig. 6 shows the profiles of the [Ar IV] $\lambda \lambda 4711,4740$ lines for high-excitation $\mathrm{PNe}$ with a stellar temperature of $150000 \mathrm{~K}$ under the assumption of outwardly decreasing and increasing density distributions. In the two sorts of density structures, the relations between the two line profiles are completely the opposite. The [Ar IV] $\lambda 4711$ line has a lower critical density than the [Ar IV] 24740 line, and thus weights lower-density regions more highly. Hence, if the nebula has a negative density gradient, the [Ar IV] $\lambda 4711$ line weights the outer regions more, where the expansion velocity is larger, which produces a broader profile in the [Ar IV] $\lambda 4711$ line compared to the [Ar IV] $\lambda 4740$ line. For a similar reason, the [Ar IV] $\lambda 4711$ line has a narrower profile than the [Ar IV] $\lambda 4740$ line for an outwardly increasing density distribution. A comparison of emission line profiles for various ionic species provides tighter constraints on nebular density structure.

\subsubsection{Density variations in the velocity space}

Using the [S II] $\lambda \lambda 6731,6716$ line profiles, we calculated the density distribution in velocity space, $N_{\mathrm{e}}(v)$, for the PNe IC 2501, IC 4191, and NGC 2440 (see Sharpee et al. 2007, for the observation details). The results are shown in Fig. 7. An inspection of the figure clearly indicates that the density variations in the velocity field are small (less than $1 \mathrm{dex}$ ). However, for the same reasons discussed in Sect. 3.1.2 for $T_{\mathrm{e}}(v)$, density variations in the velocity field may have been smoothed and only represent a lower limit to the nebular density variations unless line broadening is dominated by the expansion velocity and the velocity field is monotonic along the nebular radius.

Figure 7 shows that the compact PN IC 2501 has an almost homogeneous density structure with an average density of $\sim 10^{4} \mathrm{~cm}^{-3}$. The slight increase in density at $30 \mathrm{~km} \mathrm{~s}^{-1}$ is not real considering the large errors introduced by the weakness of the line wings. IC 4191 is a compact and irregular PN. Figure 7 shows that it has an asymmetrical density structure. A condensation is evident around $10 \mathrm{~km} \mathrm{~s}^{-1}$ on the red side, which has a density about twice as high as the other nebular regions. We

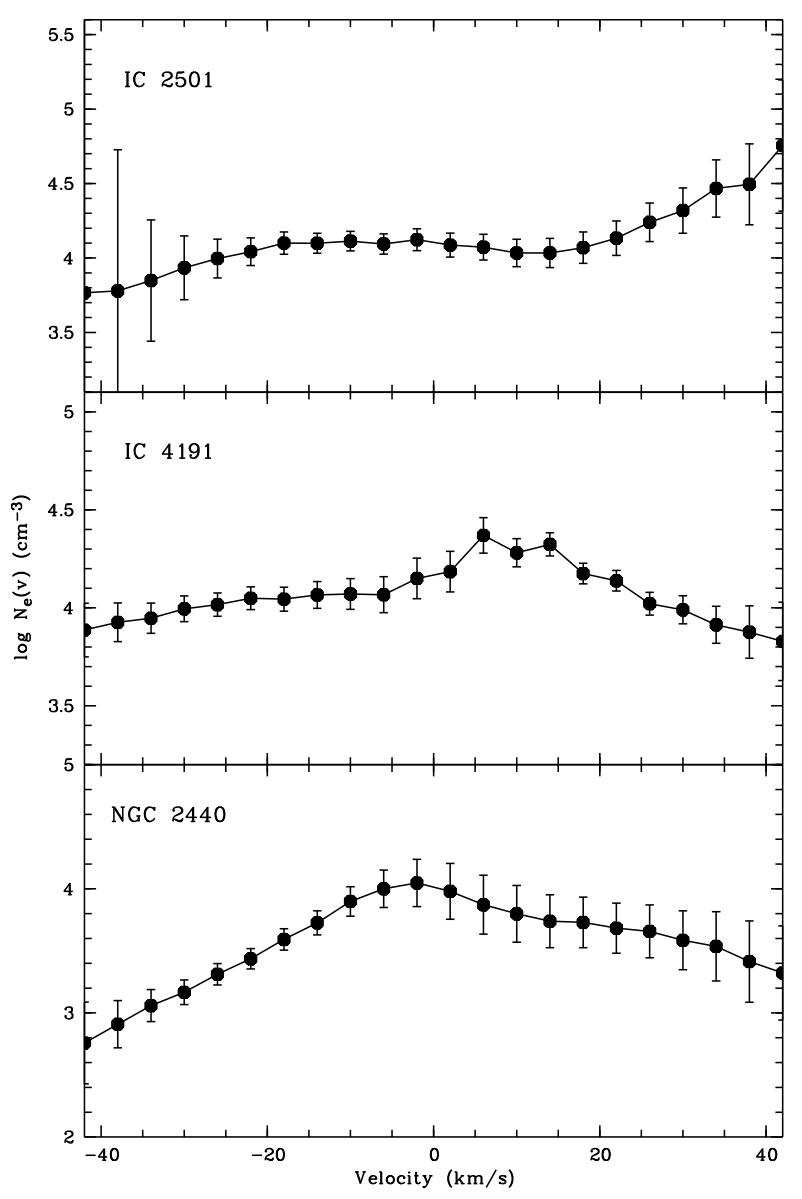

Fig. 7. Electron-density distribution in the velocity space of the PNe, IC 2501, IC 4191, and NGC 2440.

cannot find a counterpart on the blue side. NGC 2440 clearly shows a negative density gradient across the nebula. The density at the outer edge of the nebula is lower by an order of magnitude than at the centre.

We find that the [S II] $\lambda 4076$ line has a different profile from the [S II] $\lambda \lambda 6731,6716$ lines for all three PNe. However, the [S II] $\lambda 4076$ line was not taken into account in the current analysis because its upper level has a higher excitation energy than the [S II] $\lambda \lambda 6731,6716$, and hence we cannot separate the contribution of temperature variations to the difference between their profiles.

\subsection{Emission line profiles of IC 418}

To demonstrate how line profiles could be used to measure nebular physical conditions, we considered data for IC 418 to complete a detailed study of line profiles by attempting to match our models to observational data. The analysis of a larger number of PNe will appear in a separate paper. Highresolution echelle spectra of the PN IC 418 were obtained in 2001 with the $4 \mathrm{~m}$ Blanco telescope at Cerro Tololo InterAmerican Observatory (Sharpee et al. 2004), covering a wavelength range from 3500 to $9865 \AA$. IC 418 is a low-excitation $\mathrm{PN}[F([\mathrm{O} \mathrm{III}] \lambda 5007) / F(\mathrm{H} \beta) \sim 2]$ and has an approximativelyspherical shell. The observations were carried out using a $\sim 1^{\prime \prime}$ slit width, yielding a spectral resolution of $\sim 30000$. The spectrograph slit was aligned north-south. The central star was placed along a line perpendicular to the spectrograph slit so that the slit 
centre was roughly midway between the central star and outer edges of the nebula. A large number of ORL and CEL profiles are well resolved.

Hyung et al. (1994) constructed a photoionization model of IC 418 that can interpret the UV data fairly well. For our model, we initially took the basic parameters used by Hyung et al. 1994 (distance, properties of the central star, chemical composition, nebular geometrical structure, etc.), which were then adjusted slightly to achieve an optimal fit to optical observations. The density distribution of IC 418 is a topic of ongoing debate. Hyung et al. (1994) presented that the nebula may consist of two shells, an inner high-density one and an outer lower-density shell. A conflicting conclusion was obtained by Gesicki et al. (1996) who found that the density increases smoothly outwards by fitting the surface-brightness profiles for $\mathrm{H} \beta$, [O III] $\lambda 5007$, and [N II] 26584 . Our model assumed a single shell with a homogeneous density of $N_{\mathrm{H}}=10^{4} \mathrm{~cm}^{-3}$. Below we show that this issue can be settled by comparing the profiles of density diagnostic lines.

Once the photoionization model provides satisfactory fits to the observed integrated line intensities, we calculate the line profiles by assuming a velocity field and compare the predicted results with the observed ones. For the comparison, we used a number of strong lines from different ionic species, including $\mathrm{H}$ I, He I, [S II], [N II], [O I], [O II], [O III], and [Ne III]. The observed and predicted line profiles are presented by Fig. 8 . Low-ionization lines have generally broader profiles than highionization lines, which implies that the expansion velocity is increasing outwards. To reproduce the observed line profiles, the acceleration is required to increase in the outer regions, as shown in Fig. 8. For the fitting, no turbulence broadening is needed. Figure 8 shows that our model can account for most of these strong lines.

Figure 9 plots the FWHMs of the lines as a function of ionization energy, as predicted by our model and observed by Sharpee et al. (2004, see their Fig. 7). Good agreement between the predictions and the observations is achieved. The velocity field produced is in general agreement with the results of Gesicki et al. (1996), but has a larger outer velocity. Given that Gesicki et al. (1996) did not consider the [O I] line, their measured outer velocity is unlikely to be reliable. The sharply-increasing velocity in the outer regions is associated with the shock at the ionization front, which is consistent with the predictions of hydrodynamic models (e.g. Perinotto et al. 1998).

The [O II] $\lambda 7330$ line can be explained by our model, while the observed [O II] $\lambda 3726$ line is broader than the predicted one. This is unlikely to be due to temperature variations since the [N II] $\lambda \lambda 5754,6584$ doublet lines is reproduced well. The discrepancy, therefore, can be attributed to density variations within the PN. The [O II] $\lambda 3726$ line has a critical density about three times lower than that of the [O II] $\lambda 7330$ line, and thus originates in low-density regions. It follows that the outer regions have a lower density than the inner regions, in contrast to the results of Gesicki et al. (1996).

For the [O I] $\lambda 6300$ line, our model reproduces the profile in the high-velocity range well. But in the low-velocity range, the observed flux is much higher than predicted. Morisset \& Stasińska (2006a) also encountered difficulty in fitting the lowvelocity range of the [O I] $\lambda 6300$ line profile. A possible explanation is that neutral gas is plentifully abundant within the ionized regions. This conjecture was supported by Williams et al. (2003), who compared absorption- and emission-line spectra of IC 418 and found evidence that absorbing gas may be present. If we enhance the $[\mathrm{O} \mathrm{I}]$ emissivity in the inner regions by a factor
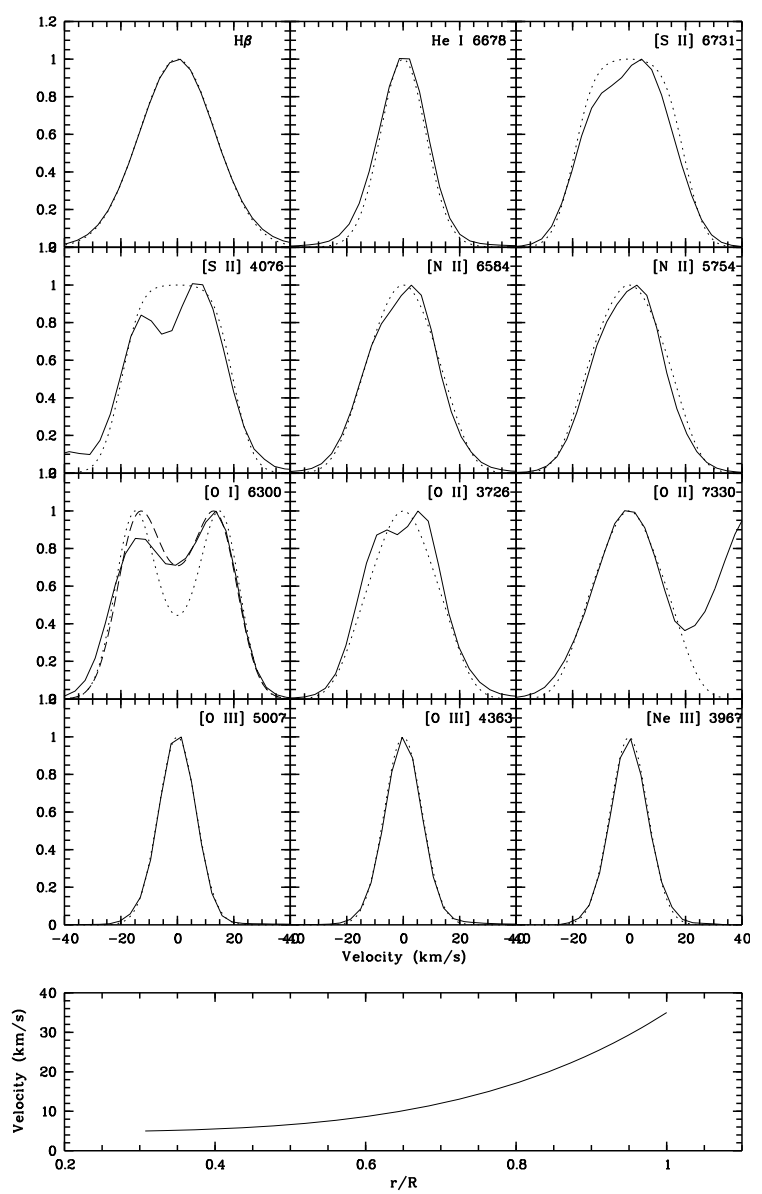

Fig. 8. Observed (solid lines) and predicted (dotted lines) strong emission lines of IC 418. The assumed velocity field is shown in the lower panel. For the [O I] $\lambda 6300$ line, the dashed line represents the predicted profile by refined emission distribution, as discussed in the text.

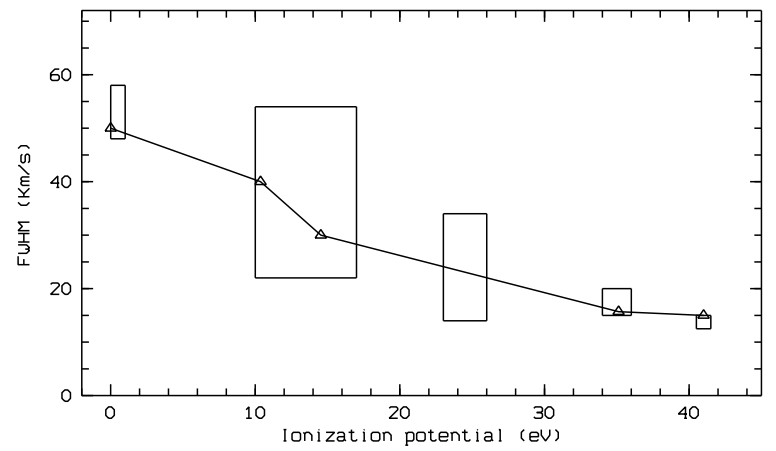

Fig. 9. FWHM as a function of the ionic species' ionization potential for the PN IC 418. The solid line with triangles is the prediction of our model. The box denotes the zone occupied by the observations of Sharpee et al. (2004).

of $\sim 1.5$, an excellent match between the predicted profile and observed one is achieved, as shown in Fig. 8.

For observations of low-excitation PN, the slit was placed in the outer edge of the $\mathrm{O}$ II regions where the projected velocity along the line of sight is quite low. Therefore, the narrow high-ionization lines, such as [O III] and [Ne III], are dominated by thermal and instrumental broadening. The [O III] $\lambda \lambda 4363,5007$ lines are therefore unsuitable for deriving temperature variations in velocity space. 


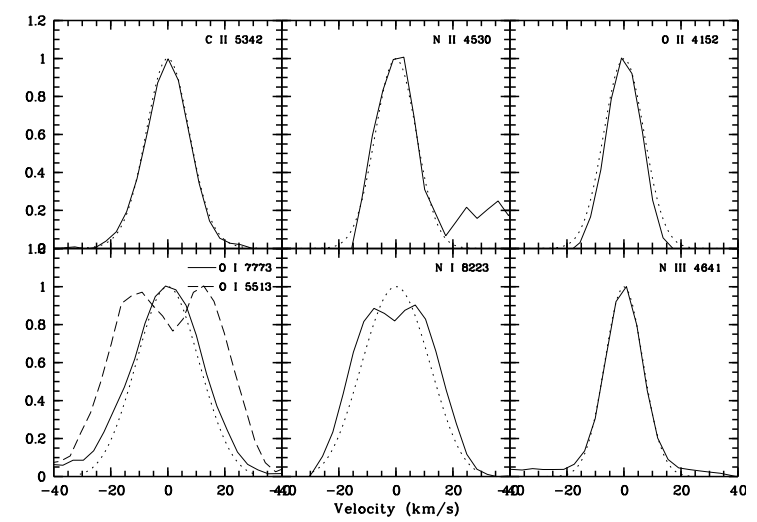

Fig. 10. Same as Fig. 8 but for recombination lines.

We compare the predicted ORL profiles with the observations. Figure 10 displays a few examples for such a comparison. Generally, our model shows good agreement with observations of the high-photoionization lines, C II, N II, O II, and $\mathrm{N}$ III. IC 418 has a small ORL/CEL abundance discrepancy $\left[\left(\mathrm{O}^{2+} / \mathrm{H}^{+}\right)_{\mathrm{ORL}} /\left(\mathrm{O}^{2+} / \mathrm{H}^{+}\right)_{\mathrm{CEL}}=1.3\right.$; Sharpee et al. 2004], and is therefore an unsuitable target to investigate the materials in which ORLs originate.

Figure 10 demonstrates that our model provides a reasonable match to the $\mathrm{O}_{\mathrm{I}} \lambda 5513$, but is unable to explain the profiles of O I $\lambda 7773$ and N I $\lambda 8223$, whose widths appear to be broader that those of the predicted lines. As pointed out by Sharpee et al. (2004), fluorescence excitation may contribute substantially to the intensity of these lines, and the radiation of the lines may originate predominantly in the outer neutral regions.

\section{Conclusions}

This paper addresses the potential of emission line profiles to probe the physical conditions of PNe. Our results show that if temperature or density variations are present within the nebulae, lines with different excitation temperatures or critical densities might show significantly different profiles even though they originate in similar ionic species. We show that line profiles provide a new way to investigate the CEL/ORL abundance-discrepancy problem. For this purpose, we require high-resolution and deep spectroscopic data. In the ideal case, the line width contributed by instrumental broadening should be smaller than that by the thermal broadening of heavy-element lines. This technical requirement corresponds to a spectral resolution higher than 75000 .

We present the electron temperatures and densities in the velocity space of a sample of PNe. No significant temperature or density variations are found. This provides a lower limit to the temperature and density inhomogeneities along the line of sight.

We attempt to reproduce the observational data of two PNe, NGC 6153 and NGC 7009, acquired by Barlow et al. (2006), which show that the [O III] $\lambda 5007$, the $[\mathrm{O}$ III] $\lambda 4363$, and the O II recombination lines have different profiles. We find that a pure photoionization model of chemically-homogeneous gas can explain the [O III] $\lambda 5007 / \lambda 4363$ profile discrepancies, but cannot explain the $[\mathrm{O}$ III] $/ \mathrm{O}$ II profile discrepancies. We thus conclude that CEL and ORL may originate in kinematically-different nebular components. Alternatively, the [O III]/O II profile discrepancies could be caused by extra heating in the outer regions. In this work, 1D computations were completed. Line profiles may significantly depend on nebular geometrical structures, as proposed by Morisset \& Stasińska (2006a). However, for lines from the same ionic species, nebular geometrical structures provide an effect on the profiles of a similar degree, and thus hardly affect our results.

We construct line profiles of the approximatively-spherical PN, IC 418. Our model can explain most of the observed line profiles. A velocity field that sharply increases outwards is revealed. We find that the [S II] and [O II] density diagnostic lines have very different profiles, which imply that large density variations are present within the PN. Our model shows that in the low-velocity regions of the [O I] $\lambda 6300$ line, the predicted flux is lower than the observed one, which indicates the existence of neutral clumps within the ionized regions. Generally, the profiles of CELs and ORLs are in good agreement. However, IC 418 is a young PN which has a small CEL/ORL abundancediscrepancy, and thus is unsuitable for studying the abundance problem. For further studies, high-quality spectroscopic observations of PNe with large CEL/ORL abundance-discrepancies should be invaluable.

Acknowledgements. I am grateful to Robert Willimas for obtaining the echelle spectra of IC 418 from their Sharpee et al. (2004) paper. I am indebted to XiaoWei Liu and Sun Kwok for valuable discussions. I also thank the referee Grazyna Stasińska for essential suggestions that helped to clarify and improve this paper significantly. The work was supported in part by a grant (Project No. HKU 7028/07P) from the Research Grants Council of Hong Kong.

\section{References}

Barlow, M. J., Hales, A. S., Storey, P. J., et al. 2006, in Planetary Nebulae, ed. M. J. Barlow, \& R. H. Méndez (Cambridge: Cambridge University Press), IAU Symp., 234, 267

Ferland, G. J., Korista, K. T., Verner, D. A., et al. 1998, PASP, 110, 761

Gesicki, K., \& Zijlstra, A. A. 2000, A\&A, 358, 1058

Gesicki, K., \& Zijlstra, A. A. 2003, MNRAS, 338, 347

Gesicki, K., Acker, A., \& Szczerba, R. 1996, A\&A, 309, 907

Gesicki, K., Acker, A., \& Zijlstra, A. A. 2003, A\&A, 400, 957

Gesicki, K., Zijlstra, A. A., Acker, A., \& Szczerba, R. 1998, A\&A, 329, 265

Hyung, S., Aller, L. H., \& Feibelman, W. A. 1994, PASP, 106, 745

Kingsburgh R. L., \& Barlow M. J. 1994, MNRAS, 271, 257

Krabbe, A. C., \& Copetti, M. V. F. 2005, A\&A, 443, 981

Liu, X.-W. 2003, in Planetary Nebulae, ed. S. Kwok, M. Dopita, \& R. Sutherland (San Francisco: ASP), IAU Symp., 209, 339

Liu, X.-W. 2006a, in Planetary Nebulae beyond the Milky Way, ed. J. Walsh, L. Stanghellini, \& N. Douglas (ESO, Garching bei München), 169

Liu, X.-W. 2006b, in Planetary Nebulae, ed. M. J. Barlow, \& R. H. Méndez (Cambridge: Cambridge University Press), IAU Symp., 234, 219

Liu, X.-W., Storey, P. J., Barlow, M. J., Danziger, I. J., Cohen, M., \& Bryce, M. 2000, MNRAS, 312, 585

Liu, X.-W., Barlow, M. J., Zhang, Y., Bastin, R. J., \& Storey, P. J. 2006, MNRAS, 368,1959

Morisset, C., \& Stasińska, G. 2006a, RevMaxAA, 42, 153

Morisset, C., \& Stasińska, G. 2006b, in Planetary Nebulae, ed. M. J. Barlow, \& R. H. Méndez (Cambridge: Cambridge University Press), IAU Symp., 234, 465

Morisset, C., \& Stasińska, G. 2008, RevMaxAA, 44, 171

Peimbert, M. 1967, ApJ, 150, 825

Peimbert, M., \& Peimbert, A. 2005, RevMaxAA, 23, 9

Peimbert, M., Peimbert, M., Ruiz, M. T., \& Esteban, C. 2004, ApJ, 150, 431

Perinotto, M., Kifonidis, K., Schonberner, D., \& Marten, H. 1998, A\&A, 332, 1044

Rubin, R. H., Bhatt, N. J., Dufour, R. J., et al. 2002, MNRAS, 334, 777

Ruiz, M. T., Peimbert, A., Peimbert, M., \& Esteban, C. 2003, ApJ, 595, 247

Sharpee, B., Williams, \& R., Baldwin, J. A. 2004, ApJ, 615, 323

Sharpee, B., Zhang, Y., Willams, R., et al. 2007, ApJ, 659, 1265

Stasińska, G., \& Szczerba, R. 2001, A\&A, 379, 1024

Viegas, S. M., \& Clegg, E. S. 1994, MNRAS, 271, 993

Williams, R., Jenkins, E. B., Baldwin, J. A., \& Sharpee, B. 2003, PASP, 115, 178

Zhang, Y., Liu, X.-W., Wesson, R., et al. 2004, MNRAS, 351, 935

Zhang, Y., Liu, X.-W., Liu, Y., \& Rubin, R. H. 2005a, MNRAS, 358, 457

Zhang, Y., Rubin, R. H., \& Liu, X.-W. 2005b, RevMaxAA, 23, 15 